\title{
Diagnóstico diferencial entre nevus y melanoma en Atención Primaria, ¿cuándo biopsiar?
}

\author{
D. Caballos Villar, J. Hernández Nuñez*, F. Ledesma Martín-Pintado**, \\ R. RuIz RAMOS*** \\ MIR de $3^{\circ}$ año de MF y C. Centro de Salud de Las Rozas. Madrid. *MIR de $3^{\circ}$ año de MF y $C$. \\ Centro de Salud de Pozuelo I. Madrid. **MIR de 3o año de MF y C. Centro de Salud de \\ Majadahonda. Madrid. ***Médico de Familia. Tutora de MIR de MF y C. Coordinadora del \\ Centro de Salud de Las Rozas. Madrid. Unidad docente de Medicina de Familia y Comunita - \\ ria. Área 6a NSALUD Madrid
}

\section{RESUMEN}

No se han descubierto evidencias consistentes a favor de las reglas de valoración clínica del $A B C D$ $y$ de los 7 puntos, como herramientas útiles para decidir si se debe hacer una biopsia de lesiones compatibles con nevus o melanoma.

Palabras clave: Melanoma. Nevus. Biopsia.
Differential diagnosis between nevi and melano ma in Primary Health Care, when a biopsy is in dicated?

\section{ABSTRACT}

To decide when to perform a biopsy for histopat hological evaluation of skin lesions which could be nevi or melanoma, the usefulness of $A B C D$ and re vised seven-point checklists is not well established.

Key words: Melanoma. Nevi. Biopsy.

\section{PREGUNTA ESTRUCTURADA}

¿Son útiles las reglas de diagnóstico clínico (ABCD y de los 7 puntos) para decidir cuándo hacer una biopsia ante una lesión pigmentada que plantea el diagnóstico diferencial de nevus y melanoma?

\section{LA BÚSQUEDA}

Con el fin de tratar de localizar una revisión sistemática sobre este tema se realizó la siguiente búsqueda: "(Nevi OR mole) AND melanoma": en Cochrane Library (http://www.obgyn.net/cochrane.asp) con búsqueda booleana, en agosto de 2001 .

Con el fin de tratar de localizar otros estudios de interés al respecto, se hizo una búsqueda comple- mentaria en Medline, con la siguiente estrategia:

Se seleccionó finalmente el estudio referido abajo por tratarse de una revisión sistemática que, cuando menos, contaba con una búsqueda mucho más exhaustiva que la que podríamos haber realizado nosotros.

\section{EL ESTUDIO}

Se trata de una revisión sistemática ${ }^{1}$ en la que los autores hicieron una búsqueda en Medline(19661996) con los MeSH (Medical Subject Headings): melanoma AND skin neoplasms combinados con physical examination, sensitivity, specificity, observer variation, mass screenning, and self examination.

Se realizó con revisores independientes, se hizo una selección de artículos por su calidad con

Aceptación: 18-11-01 
criterios explícitos, aceptando los artículos con un nivel de evidencia $\mathrm{C}$ o superior (criterios de Sackett y Goldsmith $)^{2}$ que se mencionan a continuación:

-Grado A: comparación ciega e independiente de un signo o síntoma con el "patrón oro" de diagnóstico, entre un número elevado de pacientes consecutivos con sospecha de padecer la enfermedad buscada.

- Grado B: comparación ciega e independiente de un signo o síntoma con el "patrón oro" de diagnóstico, entre un número reducido de pacientes consecutivos con sospecha evidente de padecer la enfermedad buscada.

\section{- Grado C:}

1. Comparación ciega e independiente de un signo o síntoma con el "patrón oro" de diagnóstico, entre un número de pacientes no consecutivos con sospecha de padecer la enfermedad buscada.

2. O bien, comparación no independiente de un signo o síntoma con el "patrón oro" de diagnóstico, entre un número de pacientes con evidente sospecha de padecer la enfermedad buscada.

3. O bien, comparación no independiente de un signo o síntoma con "patrón oro" de dudosa validez.

No se evaluó la heterogeneidad. No se combinaron los datos entre unos estudios y otros.

En dos estudios se evaluaban los criterios de la American Academy of Dermatology $\mathrm{ABCD}(\mathrm{E})$ (A = Asimetría, $\mathrm{B}=$ Borde irregular, $\mathrm{C}=$ Color irregular, $\mathrm{D}=$ Diámetro mayor o igual a $6 \mathrm{~mm}, \mathrm{E}=$ Elevación de la lesión).

En tres estudios se evaluaban los criterios de los 7 puntos modificada o Glasgow score (3 criterios mayores: cambios de tamaño, forma y color; 4 criterios menores: inflamación, cambios en la sensibilidad local, presencia de costra y diámetro mayor o igual a $7 \mathrm{~mm}$; se asignan 2 puntos por cada criterio mayor y 1 punto por cada criterio menor).

El "patrón oro" fue la biopsia de las lesiones.

\section{RESULTADOS PRINCIPALES}

Se reseñan únicamente aquellos estudios en los que se aplicaba la prueba a pacientes con y sin enfermedad, en los que se podía deducir los datos de sensibilidad y especificidad. Esto ocurría sólo en dos estudios mencionados en la revisión: uno sobre el $\mathrm{ABCD}^{3}$ y otro sobre los 7 puntos $^{4}$ (Tabla I).

Para la regla del ABCD-E se consideró un test positivo cuando al menos la lesión cumplía los tres criterios B $+\mathrm{C}+\mathrm{D}$. Para la regla de los 7 puntos el test se consideró positivo si la puntuación fue de 3 o más puntos.

\section{COMENTARIOS (VALIDEZ, UTILIDAD Y APLICABILIDAD)}

\section{Validez}

-Puntos fuertes: se trata de una revisión sistemática que se hace una pregunta sobre un tema claramente definido (evaluar distintas técnicas diagnósticas para diferenciar nevus y melanoma) realizándose una búsqueda sobre artículos disponibles al respecto siendo valorada la calidad de los mismos por unos criterios previamente establecidos.

-Puntos débiles: los criterios de calidad en la selección de los artículos son muy poco exigentes, lo que da lugar a la selección de trabajos de muy baja calidad. Los autores no especifican claramente la metodología seguida para hacer la selección de los artículos ni se explican los criterios de selección, que están reseñados en otro artículo diferente.

No se ha realizado una búsqueda manual, no se buscaron artículos no publicados y no queda claro si se buscaron artículos en lengua no inglesa, por lo que puede haber artículos relevantes no seleccionados.

\section{Tabla I}

\begin{tabular}{|c|c|c|c|c|c|c|}
\hline \multicolumn{7}{|c|}{ MÉTODOS DIAGNÓSTICOS DE MELANOMA } \\
\hline Fuente & $\begin{array}{c}\text { Método } \\
\text { diagnóstico }\end{array}$ & $\begin{array}{l}\text { Con/ sin } \\
\text { melanoma }\end{array}$ & $\begin{array}{l}\text { Sensibilidad } \\
\text { (IC 95\%) }\end{array}$ & $\begin{array}{l}\text { Especificidad } \\
\text { (IC 95\%) }\end{array}$ & $\begin{array}{l}\text { Coc. de prob. } \\
+(\text { IC } 95 \%)\end{array}$ & $\begin{array}{l}\text { Coc. de prob. } \\
\text { - (IC } 95 \%)\end{array}$ \\
\hline $\begin{array}{l}\text { Mc Govern y } \\
\text { Litaker }^{3}\end{array}$ & ABCD-E & $6 / 186$ & $100(54-100)$ & $98,4(95-99)$ & $62(19-170)$ & $0(0-0,5)$ \\
\hline $\begin{array}{l}\text { Healsmith } \\
\text { et } \mathrm{al}^{4}\end{array}$ & 7 puntos & $65 / 100$ & $100(94-100)$ & $37(28-46)$ & $1,6(1,4-1,9)$ & $0(0-0,2)$ \\
\hline
\end{tabular}


Los resultados no se han combinado, aunque tampoco parece razonable combinarlos dada la heterogeneidad de la calidad de los estudios y de sus resultados.

Finalmente, todos los estudios se han realizado en consultas de dermatología, por lo que los resultados deben ser valorados con mucha cautela desde Atención Primaria.

\section{Importancia de los resultados}

Depende de la probabilidad pretest de encontrar melanoma en Atención Primaria y de las características de los tests.

La probabilidad pretest para lesiones dudosas en Atención Primaria es desconocida. Los resultados de algunos trabajos realizados por médicos de familia norteamericanos sugieren una probabilidad pretest en torno al $0,4 \%{ }^{5}$. Esta tasa coincide con la probabilidad pretest entre dermatólogos cuando consideran que es poco probable que la lesión sea un melanoma. Cuando consideran que el riesgo es moderado, la probabilidad pretest para ellos asciende a un $7,7 \%$ y cuando consideran que el riesgo de que la lesión sea un melanoma es alto la probabilidad pretest asciende a un $61,7 \%^{6}$.

Sería deseable encontrar un test que incrementará la probabilidad pretest desde una tasa mínima (quizá en torno al 0,5\% en caso de lesiones que impresionan de bajo riesgo de malignidad) a una probabilidad postest de, al menos, un 3-5\%, en cuyo caso parece razonable proponer biopsia. Al mismo tiempo sería deseable encontrar un test que redujera drásticamente la probabilidad pretest para descartar la realización de biopsia.

Los resultados obtenidos sugieren que un resultado positivo con la regla ABCD partiendo de una probabilidad pretest del $0,5 \%$ daría lugar a una probabilidad postest de más del $20 \%$ de melanoma. Estos resultados parecen poco creíbles en el contexto de la Atención Primaria. Además los intervalos de confianza son amplios (ver tablas de resultados principales) por lo que la precisión es baja.

La regla de los 7 puntos, en caso de ser positiva, daría lugar a una probabilidad postest menor del $1 \%$, por lo que no parece útil tomar la decisión de realizar una biopsia.

A la hora de descartar la necesidad de realizar la biopsia se muestra aparentemente útil la regla de los 7 puntos, con un cociente de probabilidad negativo de $0(0-0,2)$. Para ello es necesario que sólo haya un criterio mayor (cambios de tamaño, color o forma), o un máximo de dos menores (inflamación, alteraciones de la sensibilidad local, costra o diámetro mayor o igual a $7 \mathrm{~mm}$ ).

\section{Aplicabilidad}

El ABCD-E tiene la ventaja de su fácil memorización. No así la regla de los 7 puntos, que puede ser algo más compleja de aplicar. Dado que los datos se muestran enormemente inciertos respecto a su validez y utilidad, cualquier reflexión relativa a la aplicabilidad de los mismos es fútil.

\section{Comentarios finales}

El melanoma es el cáncer más frecuente entre los 25 y 29 años en raza caucásica ${ }^{7}$ y uno de los más rápidamente letales.

Ante una lesión dudosa (para el paciente o para el médico) se debería:

1. Preguntar acerca de factores de riesgo (nevus congénitos, léntigo maligno, historia de nevus displásicos o melanoma, ver tabla II $)^{8}$, cambios recientes en la lesión o síntomas locales.

2. Explorar la lesión valorando simetría, bordes, coloración y diámetro mayor; y el máximo de superficie corporal buscando otras lesiones sospechosas.

Si después de todo ello persiste una duda razonable se puede biopsiar la lesión, observar la evolución periódicamente en nuestra consulta o derivar al dermatólogo.

En cualquier caso nos manejamos con gran incertidumbre al tomar decisiones en este tipo de problema en Atención Primaria. La duda, además se plantea no sólo entre nevus y melanoma. También hay que tener en cuenta otras posibilidades como nevus atípico o léntigo maligno.

\section{Tabla II}

\begin{tabular}{|c|c|}
\hline Factor de riesgo & Riesgo relativo \\
\hline $\begin{array}{l}\text { Nevus displásico, melanoma y } \\
\text { antecedentes familiares } \\
\text { de melanoma }\end{array}$ & 500 \\
\hline $\begin{array}{l}\text { Nevus displásico, no melanoma } \\
\text { previo y antecedentes familiares } \\
\text { de melanoma }\end{array}$ & 148 \\
\hline $\begin{array}{l}\text { Nevus displásico, no melanoma } \\
\text { previo ni antecedentes familiares } \\
\text { de melanoma }\end{array}$ & 7 a 27 \\
\hline Léntigo maligno & 10 \\
\hline Nevus congénito & 2 a 21 \\
\hline
\end{tabular}




\section{CONCLUSIONES}

Los estudios se han realizado en clínicas dermatológicas y en ámbito anglosajón, por lo que la validez externa, utilidad y aplicabilidad en nuestro medio es muy limitada a la hora de decidir si biopsiar o no.

Ante un paciente en el que se plantea la duda, si la lesión presenta un borde irregular, color irregular y tamaño mayor de $6 \mathrm{~mm}$, parece razonable proponer la biopsia $(\mathrm{B}+\mathrm{C}+\mathrm{D})$.

Asimismo parece razonable no biopsiar aquellas lesiones con una puntuación menor de 3 en el test de los 7 puntos.

Sin embargo, finalmente, el criterio clínico debe prevalecer sobre los resultados de los tests aplicados.

\section{AGRADECIMIENTOS}

Al Dr. Santiago Álvarez Montero* y al Dr. Fernando Caballero Martínez**, por su ayuda y estímulo para realizar este trabajo.
*Médico de Familia. Tutor extrahospitalario de MF y C. Responsable del Programa de Aprendizaje por Resolución de Problemas y Medicina Basada en Evidencias del Área $6^{\mathrm{a}}$ del INSALUD de Madrid.

**Coordinador de la Unidad Docente del Área $6^{\mathrm{a}}$ del INSALUD.

\section{Bibliografía}

1. Whited JD, Grichnik JM. Does this patient have a mole or a melanoma? JAMA 1998; 279(9): 696-701.

2. Holleman R, Simel DL. Does the clinical examination predict airflow limitation? JAMA 1995; 273(4): 313-9.

3. McGovern TW, Litaker MS. Clinical predictors of malignant pigmented lesions: a comparison of the Glasgow seven-point checklist and the American Cancer Society's ABCDs of pigmented lesions. J Dermatol Surg Oncol 1992; 18: 22-6.

4. Healsmith MF, Bourke Jf, Osborne JE, Graham-Brown RAC. An evaluation of the revised seven-point checklist for the early diagnosis of cutaneous melanoma. $\mathrm{Br} \mathrm{J}$ Dermatol 1994; 130: 48-50.

5. Limpert GH. Skin-cancer screening:a three-year experience that paid for itself. Journal of Family Practice 1995; 40 (5): 471-5.

6. Collas H, Debarrre M, De Preville PA, Courville Ph, Neveu $\mathrm{C}$, Dompmartin A, et al. Evaluation of diagnosis of pigmented cutaneous lesions and factors influencing decisions to perform excision. Ann Dermatol Venereol 1999; 126: 494-9.

7. Ries LAG, Hankey BF, Miller BA, Hartman AM, Edwards BK. Cancer Statistics Review 1973-1988. Bethesda, Md:National Cancer Institute 1991.

8. Rhodes AR, Weinstock MA, Fitzpatrick TB, Mihm MC, Sober AJ. Risk for cutaneous melanoma. A practical method of recognizing predisposed individuals. JAMA 1987; 258: 3146 . 